\title{
Genuine multipartite Bell inequality for device-independent conference key agreement
}

\author{
Timo Holz $\odot,{ }^{*}$ Hermann Kampermann, and Dagmar Bruß $\odot$ \\ Institut für Theoretische Physik III, Heinrich-Heine-Universität Düsseldorf, D-40225 Düsseldorf, Germany
}

(Received 31 October 2019; accepted 29 April 2020; published 29 May 2020)

\begin{abstract}
A class of genuine multipartite Bell inequalities is presented, which is designed for multipartite deviceindependent (DI) quantum key distribution (QKD). We prove the classical bounds of this inequality and compute DI secret-key rates based on its violation. To this end, semidefinite programming techniques are employed and extended to the multipartite case. Our Bell inequality is a nontrivial generalization of the Clauser-HorneShimony-Holt inequality. For DIQKD, we suggest an honest implementation for any number of parties and study the effect of noise on achievable conference key rates.
\end{abstract}

DOI: 10.1103/PhysRevResearch.2.023251

\section{INTRODUCTION}

Among a variety of quantum technology applications [1-3], quantum key distribution (QKD) is one of the most prominent concepts, in particular for multiple parties in a quantum network [4]. Early proposed QKD protocols [5-7] have high demands on experimental assumptions which are difficult to guarantee. Device-independent (DI) QKD aims at establishing a secret key without making detailed assumptions about the inner working processes of the quantum devices [8-12].

The security of DIQKD protocols is based on a loopholefree violation of a Bell inequality [11-18]. A connection between the DI secret-key rate and the violation of the associated Clauser-Horne-Shimony-Holt (CHSH) inequality [19] was established in Refs. [11,12] for the bipartite setting. In Ref. [18], a protocol to generate a secret key among $n$ parties, called DI conference key agreement (DICKA) was introduced, which relies on the violation of the parity-CHSH inequality. Hereby, nonlocality is certified via an effective Bell test of two parties depending on the measurement results of the remaining ones.

Not all multipartite Bell inequalities are suitable for DIQKD because measurements and quantum resources are required that allow a sufficiently large Bell-inequality violation and at the same time provide highly correlated measurement results among all parties. Moreover, at least one party has to use one measurement for key generation and for the Bell test, to detect a potential tampering of the devices. Achieving these requirements simultaneously should therefore be guaranteed by the very structure of the Bell inequality. This constraint disqualifies several known Bell inequalities as a viable option for a Bell test in DIQKD with certain quantum states. For instance, the archetypical $n$-partite Greenberger-HorneZeilinger (GHZ) state [20] can maximally violate the $n$ -

\footnotetext{
*holzt@uni-duesseldorf.de

Published by the American Physical Society under the terms of the Creative Commons Attribution 4.0 International license. Further distribution of this work must maintain attribution to the author(s) and the published article's title, journal citation, and DOI.
}

partite Mermin-Ardehali-BelinskiǐKlyshko (MABK) inequality [21-23] and also the Bell inequality most recently introduced in Ref. [24]. However, as proven in Ref. [4], perfectly correlated measurement results with the $n$-GHZ state can only be obtained if and only if all parties measure in the $\sigma_{z}$ eigenbasis, which then excludes maximum violation of the Bell inequalities in Refs. [21-24] (see Ref. [25]).

In this work, we specifically design a class of multipartite Bell inequalities that fulfills the aforementioned conditions. We prove the classical bounds of this inequality and discuss some features of it, in particular how to obtain a large Bellinequality violation. To demonstrate the usefulness of our Bell inequality, we quantify achievable conference key rates based on its violation. For this, we use the approach of Ref. [13], which employs the Navascués-Pironio-Acín (NPA) hierarchy [26,27], together with a multipartite constraint. We propose an honest implementation for a multipartite DIQKD protocol and briefly discuss how noise affects the achievable asymptotic DI secret conference key rates.

\section{A GENUINE MULTIPARTITE BELL INEQUALITY}

We impose the following condition on the Bell test: Its structure has to be such that it allows to simultaneously yield highly correlated measurement results and sufficiently large Bell-inequality violation for certain quantum states. These are crucial ingredients in any DIQKD protocol.

Consider a setup of $n$ parties, called Alice and $\mathrm{Bob}^{(j)}$ for $j \in\{2, \ldots, n\}=:[n]$ (cf. Fig. 1). Let each party measure two dichotomic observables $A_{x}$ and $B_{y^{(j)}}^{(j)}$, with inputs $x, y^{(j)} \in$ $\{0,1\}$. We define a set that contains all ordered possibilities to choose $l$ out of the labels $\{2, \ldots, n\}$ for the Bobs:

$$
\begin{aligned}
\mathcal{S}_{l}^{(n)}:= & \left\{\boldsymbol{\alpha}_{l}^{(n)}:=\left(\alpha_{l, 1}^{(n)}, \ldots, \alpha_{l, l}^{(n)}\right) \mid \alpha_{l, j}^{(n)}<\alpha_{l, j+1}^{(n)}\right. \\
& \left.\forall j \in\{1, \ldots, l-1\}, \alpha_{l, j}^{(n)} \in[n]\right\},
\end{aligned}
$$

for all $n \in \mathbb{N}, l \in\{1, \ldots, n-1\}$, with vectors $\boldsymbol{\alpha}_{l}^{(n)}$ of length $l$, whose ordered components $\alpha_{l, j}^{(n)}$ label a specific Bob; e.g., $\mathcal{S}_{2}^{(4)}=\{(2,3),(2,4),(3,4)\}$. For the sake of legibility, we also use the abbreviation

$$
B_{ \pm}^{(j)}:=\frac{1}{2}\left(B_{0}^{(j)} \pm B_{1}^{(j)}\right)
$$




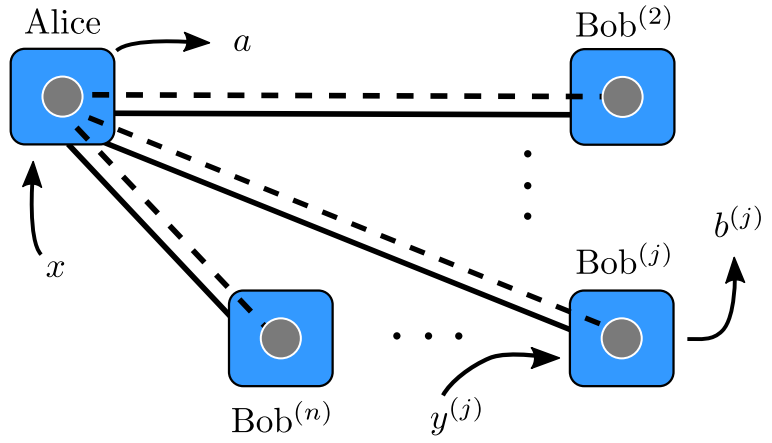

FIG. 1. A multipartite DIQKD setting, with parties Alice and $\left\{\mathrm{Bob}^{(j)}\right\}_{j=2}^{n}$. Alice distributes a multipartite quantum state via quantum channels (dashed lines). The parties communicate over classical channels (solid lines) and they perform measurements on their part of the quantum resource, specified via an input $x, y^{(j)} \in\{0,1\}$ that yields a result $a, b^{(j)} \in\{ \pm 1\}$.

Definition (genuine multipartite Bell inequality). Let $n \geqslant 3$ be an integer and $\mathcal{S}_{l}^{(n)}$ the set defined in Eq. (1).

$$
\begin{aligned}
\mathcal{B}^{(n)}:= & \left\langle A_{1} \bigotimes_{j=2}^{n} B_{+}^{(j)}\right\rangle-\delta_{\left\lfloor\frac{n}{2}\right\rfloor,\left\lceil\frac{n}{2}\right\rceil}\left\langle A_{0} \bigotimes_{j=2}^{n} B_{-}^{(j)}\right\rangle \\
& -\sum_{k=1}^{\left\lfloor\frac{n-1}{2}\right\rfloor}\left\lceil\left\langle A_{0} \otimes \sum_{\alpha_{2 k-1}^{(n)} \in \mathcal{S}_{2 k-1}^{(n)}} \bigotimes_{j=1}^{2 k-1} B_{-}^{\left(\alpha_{2 k-1, j}^{(n)}\right)}\right\rangle\right. \\
& \left.+\left\langle\sum_{\boldsymbol{\alpha}_{2 k}^{(n)} \in \mathcal{S}_{2 k}^{(n)}} \bigotimes_{j=1}^{2 k} B_{-}^{\left(\alpha_{2 k, j}^{(n)}\right)}\right\rangle\right]\left\{\begin{array}{l}
\leqslant g_{\mathrm{cl}}^{(n) \downarrow} \\
g_{\mathrm{cl}}^{(n) \uparrow}
\end{array}\right.
\end{aligned}
$$

defines a genuine multipartite Bell inequality, with upper and lower classical bounds $g_{\mathrm{cl}}^{(n) \downarrow}$ and $g_{\mathrm{cl}}^{(n) \uparrow}$, respectively.

Remember that $B_{+}^{(j)}$ and $B_{-}^{(j)}$ depend on each other [see Eq. (2)]. To make the Bell correlator more accessible, we state it for $n=3$,

$$
\mathcal{B}^{(3)}=\left\langle A_{1} B_{+}^{(2)} B_{+}^{(3)}\right\rangle-\left\langle A_{0}\left(B_{-}^{(2)}+B_{-}^{(3)}\right)\right\rangle-\left\langle B_{-}^{(2)} B_{-}^{(3)}\right\rangle,
$$

and visualize it in Fig. 2 for $n=4$.

Lemma (reduction of party number). For all $n \geqslant 2, \mathcal{B}^{(n-1)}$ is recovered from $\mathcal{B}^{(n)}$ via $B_{0}^{(n)}=B_{1}^{(n)}=\mathbb{1}$.

Proof. We have $B_{-}^{(n)}=0$; hence

$$
\bigotimes_{j=1}^{l} B_{-}^{\left(\alpha_{l, j}^{(n)}\right)}=0 \quad \forall \alpha_{l}^{(n)} \in \mathcal{S}_{l}^{(n)} \backslash \mathcal{S}_{l}^{(n-1)} .
$$
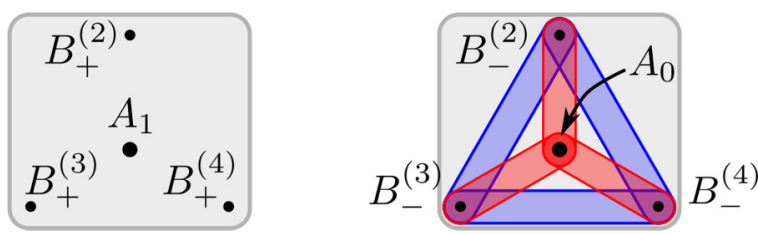

FIG. 2. Graphical representation of the correlators in the Bell inequality (3) for $n=4$, which highlights the special role of Alice and the symmetry of the inequality with respect to the Bobs. Vertices denote observables, and each hyperedge symbolizes a correlator that contains the corresponding observables.
Therefore, the sum over the set $\mathcal{S}_{l}^{(n)}$ is converted into a sum over $\mathcal{S}_{l}^{(n-1)}$. For $n$ odd, the term $\left\langle A_{0} \bigotimes_{j=2}^{n-1} B_{-}^{(j)}\right\rangle$ emerges from the sum in inequality (3) for $k=\frac{n-1}{2}$. As $B_{+}^{(n)}=\mathbb{1}$, the proof is complete.

By iteration, $\mathcal{B}^{(k)}$ is obtained from $\mathcal{B}^{(n)}$ for all $k<n$.

Theorem (classical bounds). In any classical theory, the lower and upper bounds on $\mathcal{B}^{(n)}$ are given by

$$
g_{\mathrm{cl}}^{(n) \uparrow}=-\left(2^{n-1}-1\right) \quad \text { and } \quad g_{\mathrm{cl}}^{(n) \downarrow}=1 \quad \forall n \in \mathbb{N} .
$$

Note that the upper bound is independent of $n$. See Appendix A for the analytical proof, whose idea is to consider all classical deterministic strategies, which can be significantly reduced by exploiting the invariance of $\mathcal{B}^{(n)}$ under arbitrary relabeling of Bobs.

Here, some remarks are due. First, note that for $n=2$, $\mathcal{B}^{(n)}$ and the classical bounds reproduce the $\mathrm{CHSH}$ inequality (normalized with a factor $\frac{1}{2}$ ). Furthermore, the parity-CHSH inequality [18] is a subclass of our family of Bell inequalities (see Appendix B for details), which is obtained via the choice $B_{0}^{(j)}=B_{1}^{(j)}=: B^{(j)}$ for all $j \geqslant 3$, i.e., by reducing the number of measurements to one for $\mathrm{Bob}^{(3)}$ to $\mathrm{Bob}^{(n)}$. Also, note that the lower classical bound on $\mathcal{B}^{(n)}$ is close to the algebraic minimum of $-2^{n-1}$. As we did not find a way to violate the lower bound, a violation of the Bell inequality (3) refers to the upper bound throughout this paper. Beyond that, a characterization of the maximum Bell value achievable with quantum correlations, the Tsirelson bound $g_{\mathrm{qm}}^{(n)}$ [28], is desirable. However, there is no general approach known that yields a tight Tsirelson bound for an arbitrary Bell inequality, as mentioned in Ref. [29]. An upper bound on the Tsirelson bound can be found by using the NPA hierarchy [27]. Usually, this procedure is numerically expensive, which is why we only calculate this bound for the first nontrivial odd- and even-numbered case, i.e., for $n \in\{3,4\}$ :

$$
g_{\mathrm{qm}}^{(3)}=1.5 \text { and } g_{\mathrm{qm}}^{(4)} \approx 1.5539 .
$$

These bounds are tight within numerical precision.

\section{ON THE CONSTRUCTION OF $\mathcal{B}^{(n)}$}

The Bell inequality (3) is particularly designed for the state $\left|\mathrm{GHZ}_{n}\right\rangle=\frac{1}{\sqrt{2}}\left(|0\rangle^{\otimes n}+|1\rangle^{\otimes n}\right)$, such that a maximum violation can be achieved with $A_{0}=\sigma_{z}$. To assess this statement, we start by recalling the notation we already introduced in Ref. [25]. Let $\mathbb{F}_{2}=\{0,1\}$ denote the finite field with two elements, which allows us to define the vector space $\mathbb{F}_{2}^{n}$ of bit strings of length $n$. Let further $\mathcal{P}_{n}$ denote the $n$-qubit Pauli group. We define the stabilizer group

$$
\left.\mathcal{S}:=\left\{S \in \mathcal{P}_{n}|S| \mathrm{GHZ}_{n}\right\rangle=\left|\mathrm{GHZ}_{n}\right\rangle\right\}
$$

of the $n$-GHZ state $\chi_{n}=\left|\mathrm{GHZ}_{n}\right\rangle\left\langle\mathrm{GHZ}_{n}\right|$. The group $\mathcal{S}$ is generated by the $n$ independent operators

$$
\begin{aligned}
G_{1} & :=\sigma_{x}^{\otimes n}, \quad \text { and for all } j \in[n]: \\
G_{j} & :=\bigotimes_{i=1}^{j-2} \mathbb{1}_{2}^{(i)} \otimes \sigma_{z}^{(j-1)} \otimes \sigma_{z}^{(j)} \otimes \bigotimes_{i=j+1}^{n} \mathbb{1}_{2}^{(i)},
\end{aligned}
$$


where the superscript denotes the corresponding subsystems. In general, the projector of any stabilizer state can be written as the normalized sum of all of its stabilizer operators [30,31]. We obtain for $\chi_{n}$ with $s:=\left(s_{1}, \ldots, s_{n}\right) \in \mathbb{F}_{2}^{n}$ the representation

$$
\chi_{n}=\frac{1}{2^{n}} \sum_{s \in \mathbb{F}_{2}^{n}}\left(\sigma_{x}^{s_{1}}\right)^{\otimes n}\left(\sigma_{z}^{s_{2}} \otimes \sigma_{z}^{s_{2}+s_{3}} \otimes \cdots \otimes \sigma_{z}^{s_{n-1}+s_{n}} \otimes \sigma_{z}^{s_{n}}\right) .
$$

This sum consists of $2^{n}$ individual terms, where $2^{n-1}$ of them contain only $\sigma_{z}$ and identity operators (namely, those with $s_{1}=0$ ), while the other $2^{n-1}$ ones consists of only Pauli $\sigma_{x}$ and $\sigma_{y}$ operators. The weight of such operators is given by the number of nontrivial Pauli matrices it contains. For $s_{1}=1$, the operators always have full weight, while for $s_{1}=0$ the weight of the operators is always an even number, but all possible combinations (with respect to the subsystems) of all even numbers $2 k \leqslant n$ of $\sigma_{z}$ occur. To obtain a large quantum value with the $n$-GHZ state, we aim to gain a contribution from as many operators as possible from the representation in Eq. (10). To quantify this, recall that Pauli matrices are traceless and that their product is given by

$$
\sigma_{j} \sigma_{k}=\delta_{j, k} \mathbb{1}_{2}+i \sum_{l=1}^{3} \epsilon_{j k l} \sigma_{l},
$$

where $\delta_{j, k}$ and $\epsilon_{j k l}$ denote the Kronecker delta and the LeviCivita tensor, respectively. As we require $A_{0}=\sigma_{z}$ and because of Eq. (11) the expression

$$
\operatorname{tr}\left[A_{0} \bigotimes_{j \in \mathcal{I}} B_{-}^{(j)} \sum_{s \in \mathbb{F}_{2}^{n}, s_{1}=1}\left(\sigma_{x} \sigma_{z}^{s_{2}} \otimes \cdots \otimes \sigma_{x} \sigma_{z}^{s_{n}}\right)\right]
$$

vanishes for any index subset $\mathcal{I} \subseteq\{2, \ldots, n\}$ for all $s$ with $s_{1}=1$. The counterpart of expression (12) for $s_{1}=0$, however, is nonvanishing if the observables have an even weight. The same argument can be done for the corresponding expression without an observable of Alice. As all possible even-weighted combinations occur in the $n$-GHZ state (10), we include all such possible combinations of observables with respect to the parties in our Bell inequality (3), which leads to the sum $\sum_{k=1}^{\left\lfloor\frac{n-1}{2}\right\rfloor}(\cdots)$. The expression $-\delta_{\left\lfloor\frac{n}{2}\right\rfloor,\left\lceil\frac{n}{2}\right\rceil}\left\langle A_{0} \bigotimes_{j=2}^{n} B_{-}^{(j)}\right\rangle$ is included due to a fundamental difference between the odd- and even-numbered $n$-GHZ state. For $n$ even, the operator $\sigma_{z}^{\otimes n}$ occurs in the GHZ-state representation in Eq. (10), while for $n$ odd, this is not the case. Finally, since operators with $s_{1}=1$ have full weight, we include one additional expectation value in the Bell inequality that contains observables of all parties, hence the first term in our Bell inequality (3).

\section{OPTIMAL MEASUREMENTS}

Using the structure of the Bell expression $\mathcal{B}^{(n)}$, one can derive the optimal measurement settings given the $n$-GHZ state is measured (see Appendix $\mathrm{C}$ for details). They can be chosen to be in the $\sigma_{z}-\sigma_{x}$ plane of the Bloch sphere, in detail:

$$
\begin{aligned}
& A_{0}=\sigma_{z}, \quad B_{0}^{(j)}=\sin (\theta) \sigma_{x}+\cos (\theta) \sigma_{z}, \\
& A_{1}=\sigma_{x}, \quad B_{1}^{(j)}=\sin (\theta) \sigma_{x}-\cos (\theta) \sigma_{z},
\end{aligned}
$$

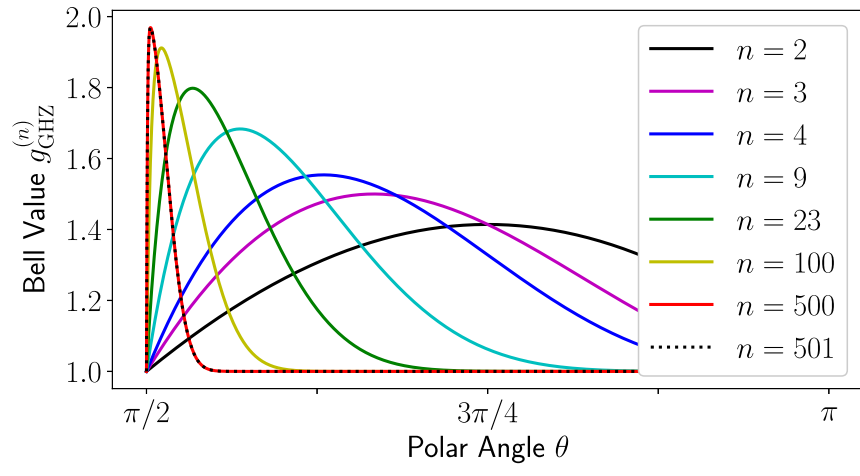

FIG. 3. Achievable Bell value $g_{\mathrm{GHZ}}^{(n)}$ according to Eqs. (14) as a function of the polar angle $\theta$ for various numbers $n$.

for all $j \in[n]$, where the optimal value of the polar angle $\theta$ depends on the number of parties, $n$. Note that, due to the symmetry of the Bell correlator and the target state, $\theta$ does not depend on $j$. This choice allows a straightforward calculation of the Bell value achievable with the $n$-GHZ state, which reads

$$
\begin{aligned}
& g_{\mathrm{GHZ}}^{(n, \text { odd })}=1-(1+\cos (\theta))^{n-1}+\sin ^{n-1}(\theta), \\
& g_{\mathrm{GHZ}}^{(n, \text { even })}=1-(1+\cos (\theta))^{n-1}+\frac{\cot (\theta / 2) \sin ^{n}(\theta)}{1+\cos (\theta)} .
\end{aligned}
$$

Table I displays some quantities of interest for $n \leqslant 7$. For any number of parties, the relation in Eqs. (14) can be numerically optimized with respect to $\theta$, which is visualized in Fig. 3. For finite $n$, every Bob has two distinct measurement settings and in the asymptotic limit we find $\lim _{n \rightarrow \infty} g_{\mathrm{GHZ}}^{(n)}=2$ and $\lim _{n \rightarrow \infty} \theta^{(n)}=\frac{\pi}{2}$.

From Table I, we notice that the Bell value $g_{\mathrm{GHZ}}^{(n)}$ for $n \in\{3,4\}$ coincides with the Tsirelson bound in Eqs. (7). Due to the symmetry and construction of the Bell inequality, we conjecture that this holds for general $n$. If this is true, finding the Tsirelson bound to our Bell inequality boils down to a simple optimization over the parameter $\theta$ in Eqs. (14). To conclude this discussion, consider the Bell inequality for $n=3$ parties. States of the form $\rho=\rho_{A B^{(2)}} \otimes \rho_{B^{(3)}}$ do not allow to exceed the Tsirelson bound for $n=2$ parties, which one can verify - either analytically or via the NPA hierarchy-by taking all classical deterministic strategies for $\mathrm{Bob}^{(3)}$ into account. Thus, $\sqrt{2}$ is a Svetlichny bound [32]

TABLE I. Maximum Bell value $g_{\mathrm{GHZ}}^{(n)}$ achievable with $n$-GHZ state [cf. Eqs. (14)], the ratio of $g_{\mathrm{GHZ}}^{(n)}$ and $g_{\mathrm{GHZ}}^{(n-1)}$, and the corresponding polar angle $\theta$ for all Bobs. The quantum-to-classical ratio is given by $g_{\mathrm{GHZ}}^{(n)}$, as $g_{\mathrm{cl}}^{(n) \downarrow}=1$ for all $n$. The values are rounded to the fourth decimal place.

\begin{tabular}{lccc}
\hline \hline $\mathcal{B}^{(n)}$ & $g_{\mathrm{GHZ}}^{(n)}$ & $g_{\mathrm{GHZ}}^{(n)} / g_{\mathrm{GHZ}}^{(n-1)}$ & $\theta$ \\
\hline $\mathcal{B}^{(2)}$ & $\sqrt{2} \approx 1.4142$ & & $\frac{3 \pi}{4} \approx 2.3562$ \\
$\mathcal{B}^{(3)}$ & 1.5 & $\frac{3}{2 \sqrt{2}} \approx 1.0607$ & $\frac{2 \pi}{3} \approx 2.0944$ \\
$\mathcal{B}^{(4)}$ & 1.5539 & 1.0359 & 1.9786 \\
$\mathcal{B}^{(5)}$ & 1.5926 & 1.0249 & 1.9106 \\
$\mathcal{B}^{(6)}$ & 1.6224 & 1.0187 & 1.8650 \\
$\mathcal{B}^{(7)}$ & 1.6464 & 1.0148 & 1.8318 \\
\hline \hline
\end{tabular}


which can certify genuine tripartite entanglement. Likewise, one observes that states of the form $\rho=\rho_{A} \otimes \rho_{B^{(2)} B^{(3)}}$ cannot violate the classical bound. Beyond the tripartite case, we have numerical indication for analogous statements concerning biseparable splits (cf. Sec. VII).

\section{BOUNDING EVE'S GUESSING PROBABILITY}

Finally, we want to apply our Bell inequality (3) for DIQKD. As preparation, we briefly describe how to obtain a lower bound on the DI conference key rates. We focus on asymptotic secret-key rates and assume that quantum devices behave according to the independent and identically distributed (i.i.d.) assumption. Let $\mathcal{G}^{(n)}$ denote the Bell operator corresponding to our Bell inequality (3), i.e., $\mathcal{B}^{(n)}=$ $\operatorname{tr}\left(\mathcal{G}^{(n)} \rho_{A \boldsymbol{B}}\right)$, where $\rho_{A \boldsymbol{B}}:=\rho_{A B^{(2)} \ldots B^{(n)}}$ represents the quantum state shared among all parties. Let Alice use measurement input $x=0$ for raw key generation and define $\boldsymbol{B}_{\boldsymbol{y}}:=$ $\left(B_{y^{(2)}}^{(2)}, \ldots, B_{y^{(n)}}^{(n)}\right)$. Eve's guessing probability $P_{\mathrm{g}}(\boldsymbol{a} \mid \mathcal{E})$ about Alice's $A_{0}$-measurement results $\boldsymbol{a}$ conditioned on her information $\mathcal{E}$ can be upper bounded by a function $f$ of the observed Bell violation $g_{\text {obs }}^{(n)}$. For fixed $g_{\text {obs }}^{(n)}$, it amounts to the solution of the semidefinite program (SDP) $[13,27,33]$

$$
\begin{aligned}
& \max _{\rho_{A \boldsymbol{B}}, A_{x}, \boldsymbol{B}_{\boldsymbol{y}}} \operatorname{tr}\left(A_{0} \rho_{A \boldsymbol{B}}\right) \\
& \text { subject to } \operatorname{tr}\left(\mathcal{G}^{(n)} \rho_{A \boldsymbol{B}}\right)=g_{\text {obs }}^{(n)} .
\end{aligned}
$$

For classical-quantum states $\rho_{A \mathcal{E}}$, the guessing probability is connected to the quantum min-entropy via $H_{\min }(\boldsymbol{a} \mid \mathcal{E})=-\log _{2} P_{\mathrm{g}}(\boldsymbol{a} \mid \mathcal{E})$ [34], from which we obtain a lower bound on the DI asymptotic secret-key rate [13], $r_{\infty, n}^{\mathrm{SDP}} \geqslant-\log _{2} f\left(g_{\text {obs }}^{(n)}\right)-h(Q)$, where $h(p):=-p \log _{2}(p)-$ $(1-p) \log _{2}(1-p)$ denotes the binary entropy and $Q$ the quantum bit error rate (QBER). The noisiest channel determines the QBER [4]; hence

$$
Q=\max _{j \in[n]}\left(Q_{A B^{(j)}}\right),
$$

where $Q_{A B^{(j)}}$ is the QBER between Alice and $\mathrm{Bob}^{(j)}$. The bound established by the SDP (15) is valid against the most general attacks the eavesdropper can perform [13] but they are in general rather loose. Recent development promises improvement in this regard [35].

\section{DEVICE-INDEPENDENT CONFERENCE KEY AGREEMENT}

Here, we present achievable DI secret-key rates for $n$ parties with a DIQKD protocol, which we present for completeness. Let Alice have two measurement inputs $x \in\{0,1\}$ and all $\mathrm{Bob}^{(j)}$ have three inputs $y^{(j)} \in\{0,1,2\}$. The protocol includes the following steps (see also Refs. [4,36]):

(i) In every round of the protocol, the parties do the following:

State preparation. Alice produces and distributes a multipartite state $\rho_{A B}$. Since we assume an i.i.d. implementation, the source generates the same state in every round.

Measurement. There are two types of measurement rounds, key generation (type-0) and parameter estimation (type-1) measurement rounds. For type 0 , the parties choose the inputs $(x, y)=(0,2, \ldots, 2)$, and for type 1 they choose their inputs $x, y^{(j)} \in\{0,1\}$ uniformly at random. The parties use a preshared random key to agree on the type of measurement round.

(ii) Parameter estimation. The parties publicly communicate the list of bases and outcomes for type- 1 rounds and an equal amount of measurement outputs for type-0 rounds. The publicly announced data from type 1 are used to estimate the Bell value $g_{\text {obs }}^{(n)}$ of inequality (3), whereas the announced type-0 data are used to estimate the quantum bit error rate $Q$, which quantifies the asymptotic error-correction information.

(iii) Classical postprocessing. Similar to the devicedependent multipartite QKD protocol [4], an error-correction and privacy-amplification protocol is performed.

If the parties verify that their data violate our Bell inequality (3), they commence the error correction. The solution of the SDP (15) then upper-bounds Eve's guessing probability. If $g_{\mathrm{obs}}^{(n)} \leqslant g_{\mathrm{cl}}^{(n) \downarrow}$ they abort the protocol.

In an honest implementation, the quantum state $\rho_{A B}$ is the $n$-GHZ state. To minimize the error-correction information, all parties measure $\sigma_{z}$ in key generation rounds. To test for Bell-inequality violation, the parties choose observables as proposed in Eq. (13) that lead to a maximum violation. The observed correlations are taken as those that one would see for a qubit depolarizing channel affecting the subsystem $\rho_{i}$ according to

$$
\mathcal{D}_{\text {dep }}\left(\rho_{i}\right)=(1-p) \rho_{i}+\frac{p}{2} \mathbb{1}_{2},
$$

where $p \in[0,1]$ denotes the noise parameter. In this scenario, the marginal probability distribution of Alice's $A_{0}$ measurement is uniform; i.e., $\left\langle A_{0}\right\rangle=0$. Since we consider binary outcomes, we can lower bound the von Neumann entropy in terms of the guessing probability via $H(a \mid \mathcal{E}) \geqslant$ $2\left(1-P_{\mathrm{g}}(a \mid \mathcal{E})\right)[35,37]$, which in turn yields

$$
r_{\infty}^{\mathcal{B}^{(n)}} \geqslant 2\left(1-P_{\mathrm{g}}(a \mid \mathcal{E})\right)-h(Q) .
$$

Figure 4 displays the lower bound on the asymptotic DI secret-key rate, Eq. (18), as a function of the parameter $p$ of the noise model in Eq. (17). To put these key rates into perspective, we consider the same comparison as in Ref. [18], where the conference key rates are compared with multiple bipartite key rates, described by [11]

$$
r_{\infty}^{\mathrm{CHSH}} \geqslant 1-h(Q)-h\left(\frac{1+\sqrt{S^{2} / 4-1}}{2}\right),
$$

where $S$ denotes the violation of the $\mathrm{CHSH}$ inequality. For illustration, we consider the Bell state $\left|\phi^{+}\right\rangle \propto|00\rangle+|11\rangle$ under the noise model in Eq. (17), which connects $S$ with $Q$ according to $S=2 \sqrt{2}(1-2 Q)$. The QBER $Q$ as defined in Eq. (16) is related to the noise parameter via $Q=p(1-p / 2)$ for all $n$. Under the assumption that Alice cannot perform the bipartite QKD protocols with every Bob simultaneously, which can be the case in bottleneck networks (cf. Ref. [4]), the bipartite key rates get a prefactor of $(n-1)^{-1}$.

The bounds on the guessing probability in terms of SDPs are often too pessimistic. Thus, we cannot beat the analytical results of Ref. [18]. But remember that the parity-CHSH inequality is a special case of our Bell inequality. Hence, using 


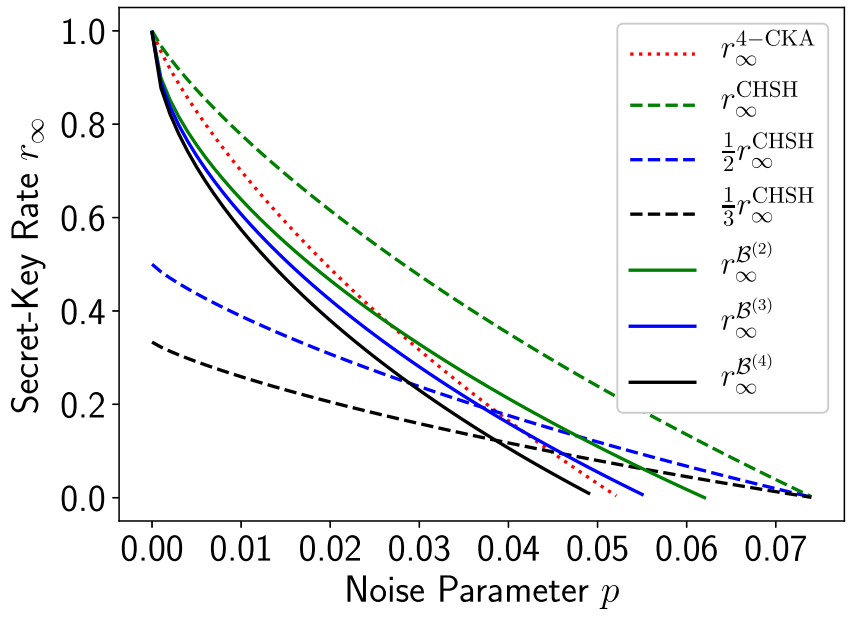

FIG. 4. Asymptotic DI secret-key rates according to Eq. (18) in dependence of the noise parameter $p$ (solid lines) for $n \in\{2,3,4\}$. In bottleneck networks and for low noise, the multipartite DIQKD protocol outperforms multiple bipartite DIQKD protocols, Eq. (19) (dashed lines). The dotted line corresponds to the analytical bounds of Ref. [18], Eq. (4) for $n=4$ in the same implementation. The comparison based on SDP rates is discussed in the main text.

SDP, the latter outperforms the former. On the scale in Fig. 4, however, this advantage is barely visible. To give an example, consider $n=3$ and noise parameters $p \in\{3,4,5\} \%$. Then, secret-key rates based on $\mathcal{B}^{(3)}$ are larger by approximately $\{1.2,3.6,16.8\} \%$. Note that the analytical bounds of Ref. [18] automatically also provide bounds via our Bell inequality. Better analytical bounds, using the full structure of our inequality, remain to be found.

\section{CONCLUSION AND OUTLOOK}

In this paper, we introduced a family of genuine multipartite Bell inequalities that is specifically tailored to the $n$-GHZ state, while maintaining the possibility to maximally violate it with $\sigma_{z}$ measurements. As argued, an application is to use this Bell inequality for a Bell test in a DIQKD protocol, because there highly correlated measurement results and maximal violation are required at the same time. We established the classical bounds of this Bell inequality and suggested measurements that lead to the maximal Bell value, given the $n$-GHZ state is measured. Finally, we calculated via semidefinite programming conference key rates based on the violation of our Bell inequality and discussed its robustness against depolarizing noise.

For future work, a more thorough study of our Bell inequality (3) is desirable. A starting point is to clarify the role of partially entangled states and the existence of associated intermediate bounds in our Bell inequality, similar to the MABK case [38]. We conjecture that the maximum Bell value $\mathcal{B}^{(n)}$ for $n$ parties with biseparable states where at most $k-1$ Bobs are entangled with Alice is determined by the maximum Bell value $\mathcal{B}^{(k)}$ for $k$ parties. In this case a Bell value larger than $\mathcal{B}^{(k)}$ is a DI witness for entanglement of at least $k+1$ parties, one of them being Alice. An important goal would be to find an analytical bound on the von Neumann entropy in terms of the violation of our Bell inequality (3). As we provided a nontrivial genuinely multipartite generalization of the $\mathrm{CHSH}$ inequality-in a similar spirit as the $n$-GHZ state represents a multipartite generalization of the Bell state-we hope that our contribution paves the way for further insight into multipartite quantum communication.

\section{ACKNOWLEDGMENTS}

The authors acknowledge support from the Federal Ministry of Education and Research BMBF (Project Q.Link.X and HQS). Funding was provided by the Deutsche Forschungsgemeinschaft (DFG, German Research Foundation) under Germany's Excellence Strategy-Cluster of Excellence Matter and Light for Quantum Computing (ML4Q) EXC 2004/1-390534769. We thank Reinhard Werner, Gláucia Murta, and Lucas Tendick for helpful discussions.

\section{APPENDIX A: PROOF OF THE THEOREM}

Here, we prove the classical upper and lower bounds of our Bell inequality, which we recall for convenience:

$$
\begin{aligned}
-\left(2^{n-1}-1\right) \leqslant & \left\langle A_{1} \bigotimes_{j=2}^{n} B_{+}^{(j)}\right\rangle-\delta_{\left\lfloor\frac{n}{2}\right\rfloor,\left\lceil\frac{n}{2}\right\rceil}\left\langle A_{0} \bigotimes_{j=2}^{n} B_{-}^{(j)}\right\rangle \\
& -\sum_{k=1}^{\left\lfloor\frac{n-1}{2}\right\rfloor}\left[\left\langle A_{0} \otimes \sum_{\alpha_{2 k-1}^{(n)} \in \mathcal{S}_{2 k-1}^{(n)}} \bigotimes_{j=1}^{2 k-1} B_{-}^{\left(\alpha_{2 k-1, j}^{(n)}\right)}\right\rangle\right. \\
& \left.+\left\langle\sum_{\boldsymbol{\alpha}_{2 k}^{(n)} \in \mathcal{S}_{2 k}^{(n)}} \bigotimes_{j=1}^{2 k} B_{-}^{\left(\alpha_{2 k, j}^{(n)}\right)}\right\rangle\right] \leqslant 1
\end{aligned}
$$

The maximal and minimal classical value is achieved for deterministic strategies. To establish the classical bounds, we thus consider the variables $A_{x}$ and $B_{y^{(j)}}^{(j)}$ for $x, y^{(j)} \in\{0,1\}$, $j \in\{2, \ldots, n\}$ to take on values from the set $\{ \pm 1\}$ and denote with the vector $\left(\boldsymbol{A}, \boldsymbol{B}^{(j)}\right)$ a strategy from the set that contains every possible combination of \pm 1 as components for this $2 n$-dimensional vector. We also define

$$
\tilde{\mathcal{B}}^{(n)}:=-\delta_{\left\lfloor\frac{n}{2}\right\rfloor,\left\lceil\frac{n}{2}\right\rceil} A_{0} \prod_{j=2}^{n} B_{-}^{(j)}-\sum_{k=1}^{\left\lfloor\frac{n-1}{2}\right\rfloor}\left[A_{0} \sum_{\alpha_{2 k-1}^{(n)} \in \mathcal{S}_{2 k-1}^{(n)}} \prod_{j=1}^{2 k-1} B_{-}^{\left(\alpha_{2 k-1, j}^{(n)}\right)}+\sum_{\alpha_{2 k}^{(n)} \in \mathcal{S}_{2 k}^{(n)}} \prod_{j=1}^{2 k} B_{-}^{\left(\alpha_{2 k, j}^{(n)}\right)}\right],
$$

such that we can write $\mathcal{B}^{(n)}=A_{1} \prod_{j} B_{+}^{(j)}+\tilde{\mathcal{B}}^{(n)}$ for the classical Bell value. We make the important observation that any strategy $\left(\boldsymbol{A}, \boldsymbol{B}^{(j)}\right)$ that leads to $A_{1} \prod_{j} B_{+}^{(j)} \neq 0$ eliminates the value of $\tilde{\mathcal{B}}^{(n)}$ as this requires that $B_{+}^{(j)} \neq 0$ (and thus $B_{-}^{(j)}=0$ ) for all $j \in[n]$. Therefore, we can maximize and minimize the expressions $A_{1} \prod_{j} B_{+}^{(j)}$ and $\tilde{\mathcal{B}}^{(n)}$ independently. This distinction into cases allows 
us to map the strategies for the maximization (minimization) of $\tilde{\mathcal{B}}^{(n)}$ from $\left(\boldsymbol{A}, \boldsymbol{B}^{(j)}\right) \in\{ \pm 1\}^{2 n}$ to $\left(A_{0}, \boldsymbol{B}_{-}^{(j)}\right)$ with $A_{0} \in\{ \pm 1\}$ and $B_{-}^{(j)}=\frac{1}{2}\left(B_{0}^{(j)}-B_{1}^{(j)}\right) \in\{ \pm 1,0\}$. For the proof we require three important properties of the binomial coefficients:

$$
\begin{array}{rlrl}
\sum_{l=0}^{n}\left(\begin{array}{l}
n \\
l
\end{array}\right) & =2^{n} & \text { (normalization), } \\
\left(\begin{array}{l}
n \\
l
\end{array}\right) & =\left(\begin{array}{c}
n-1 \\
l
\end{array}\right)+\left(\begin{array}{c}
n-1 \\
l-1
\end{array}\right) \quad \text { (Pascal triangle), } \\
\left(\begin{array}{l}
n \\
l
\end{array}\right) & =\sum_{j=0}^{l}\left(\begin{array}{c}
m \\
j
\end{array}\right)\left(\begin{array}{c}
n-m \\
l-j
\end{array}\right) \quad \text { (Chu-Vandermonde identity). }
\end{array}
$$

Note that we make use of the conventions $0 !=1$ and $\left(\begin{array}{l}n \\ l\end{array}\right)=$ $0 \forall l>n, l<0$. We divide the proof into two parts, one for the lower and one for the upper bound.

(i) Lower bound. To establish the lower classical bound, note that the minimization of $A_{1} \prod_{j} B_{+}^{(j)}$ leads only to the value of -1 . A minimization of $\tilde{\mathcal{B}}^{(n)}$, however, is given by the choice $B_{-}^{(j)}=+1$ for all $j$ and $A_{0}=+1$, as this turns every contribution in Eq. (A2) negative, in detail

$$
\begin{aligned}
\tilde{\mathcal{B}}^{(n)} & =-\delta_{\left\lfloor\frac{n}{2}\right\rfloor,\left\lceil\frac{n}{2}\right\rceil}-\sum_{k=1}^{\left\lfloor\frac{n-1}{2}\right\rfloor}\left[\left(\begin{array}{c}
n-1 \\
2 k-1
\end{array}\right)+\left(\begin{array}{c}
n-1 \\
2 k
\end{array}\right)\right] \\
& =-\delta_{\left\lfloor\frac{n}{2}\right\rfloor,\left\lceil\frac{n}{2}\right\rceil}-\sum_{k=1}^{2\left\lfloor\frac{n-1}{2}\right\rfloor}\left(\begin{array}{c}
n-1 \\
k
\end{array}\right),
\end{aligned}
$$

where we used the cardinality $\# \mathcal{S}_{l}^{(n)}=\left(\begin{array}{c}n-1 \\ l\end{array}\right)$. Via the normalization condition, Eq. (A3a), the expression above simplifies for both $n$ odd and even to $-\left(2^{n-1}-1\right)$, as claimed.

(ii) Upper bound. A maximization of $A_{1} \prod_{j} B+^{(j)}$ leads to the value of 1 , but a priori it is not clear that this is indeed the maximum possible $\mathcal{B}^{(n)}$ value. We start by counting all possible strategies for $\tilde{\mathcal{B}}^{(n)}$ and categorize them, such that we can calculate its value by a distinction of cases. There are $2 \times 3^{n-1}$ different possibilities to choose a strategy $\left(A_{0}, \boldsymbol{B}_{-}^{(j)}\right)$; however, we notice that the expression $\tilde{\mathcal{B}}^{(n)}$ in Eq. (A2) is invariant under permutation of Bobs; i.e., we only need to calculate the $\tilde{\mathcal{B}}^{(n)}$ value for a subset of strategies $\left(A_{0}, \boldsymbol{B}_{-}^{(j)}\right)$, that cannot be converted into each other by permutation of Bobs. This reduces the number of different deterministic strategies to only $n(n+1)$. As a final remark before we work through the different strategies, we note that the amount of nonzero values for the variables $B_{-}^{(j)}$ determines which summands give a nontrivial contribution to $\tilde{\mathcal{B}}^{(n)}$. To be more specific, let $q_{ \pm}$denote the amount of \pm 1 values in the strategy $\left(A_{0}, \boldsymbol{B}_{-}^{(j)}\right)$, and let $q_{+}+q_{-}=: q \leqslant n-1$ be the amount of nonzero $B_{-}^{(j)}$ values. Due to the permutational invariance of $\tilde{\mathcal{B}}^{(n)}$ we order without loss of generality the strategy $\left(A_{0}, \boldsymbol{B}_{-}^{(j)}\right)$ such that $B_{-}^{(j)}=0$ for all $j>q+1$. Then, every product in Eq. (A2) associated to a label $\boldsymbol{\alpha}_{l}^{(n)} \in \mathcal{S}_{l}^{(n)} \backslash \mathcal{S}_{l}^{(q+1)}$ vanishes, as it contains at least one $\mathrm{Bob}^{(j)}$ with $B_{-}^{(j)}=0$. This converts the sum over the set $\mathcal{S}_{l}^{(n)}$ into a sum over the set $\mathcal{S}_{l}^{(q+1)}$ of cardinality $\left(\begin{array}{l}q \\ l\end{array}\right)$. The expression $A_{0} \prod_{j=2}^{n} B_{-}^{(j)}$ always vanishes for $q<n-1$.

(a) $q \leqslant n-1, q=q_{ \pm}$. For these cases, $B_{-}^{(j)}=B_{-}^{(k)}$ holds for all $j, k \in\{2, \ldots, q+1\}$. Applying this strategy yields

$$
\begin{aligned}
\tilde{\mathcal{B}}^{(n)}= & -\delta_{\left\lfloor\frac{n}{2}\right\rfloor,\left\lceil\frac{n}{2}\right\rceil} \delta_{n-1, q} A_{0}( \pm 1)^{n-1} \\
& -\sum_{k=1}^{\left\lfloor\frac{n-1}{2}\right\rfloor}\left[A_{0} \sum_{\alpha_{2 k-1}^{(q+1)} \in \mathcal{S}_{2 k-1}^{(q+1)}}( \pm 1)^{2 k-1}+\sum_{\alpha_{2 k}^{(q+1)} \in \mathcal{S}_{2 k}^{(q+1)}}( \pm 1)^{2 k}\right] \\
= & -\delta_{\left\lfloor\frac{n}{2}\right\rfloor,\left\lceil\frac{n}{2}\right\rceil} \delta_{n-1, q} A_{0}( \pm 1)^{n-1} \\
& -\sum_{k=1}^{\left\lfloor\frac{n-1}{2}\right\rfloor}\left[ \pm A_{0}\left(\begin{array}{c}
q \\
2 k-1
\end{array}\right)+\left(\begin{array}{c}
q \\
2 k
\end{array}\right)\right] .
\end{aligned}
$$

To proceed, let $n$ be an odd integer; hence $\delta_{\left\lfloor\frac{n}{2}\right\rfloor,\left\lceil\frac{n}{2}\right\rceil}=0$. Then, the best Alice can do is to choose her variable $A_{0} \in\{ \pm 1\}$ such that the sum is minimized, because of the global minus sign in Eq. (A5). Exploiting identity (A3b) leads to

$$
\tilde{\mathcal{B}}^{(n)}=-\sum_{k=1}^{\frac{n-1}{2}}\left[-\left(\begin{array}{c}
q-1 \\
2 k-2
\end{array}\right)+\left(\begin{array}{c}
q-1 \\
2 k
\end{array}\right)\right]
$$

where the only nonvanishing term is $\left(\begin{array}{c}q-1 \\ 0\end{array}\right)$ and thus results in $\tilde{\mathcal{B}}^{(n)}=1$. For $n$ even, we can make a similar argument. Choosing the value for $A_{0}$ that maximizes the total expression leads us to

$$
\begin{aligned}
\tilde{\mathcal{B}}^{(n)} & =\delta_{n-1, q}+\left(\begin{array}{c}
q-1 \\
0
\end{array}\right)-\left(\begin{array}{l}
q-1 \\
n-2
\end{array}\right) \\
& =\delta_{n-1, q}+1-\delta_{n-1, q}=1 .
\end{aligned}
$$

(b) $q_{+}+q_{-}=q \leqslant n-1, q_{ \pm} \geqslant 1$. For the remaining cases, at least one variable $B_{-}^{(j)}$ is +1 and at least one is -1 . 
From Eq. (A2) we obtain with this strategy

$$
\begin{aligned}
\tilde{\mathcal{B}}^{(n)}= & (-1)^{q_{-}+1} \delta_{\left\lfloor\frac{n}{2}\right\rfloor,\left\lceil\frac{n}{2}\right\rceil} \delta_{n-1, q} A_{0} \\
& -\sum_{k=1}^{\left\lfloor\frac{n-1}{2}\right\rfloor}\left[A_{0} \sum_{r=0}^{2 k-1}(-1)^{r}\left(\begin{array}{c}
q_{+} \\
2 k-1-r
\end{array}\right)\left(\begin{array}{c}
q_{-} \\
r
\end{array}\right)\right. \\
& \left.+\sum_{r=0}^{2 k}(-1)^{r}\left(\begin{array}{c}
q_{+} \\
2 k-r
\end{array}\right)\left(\begin{array}{c}
q_{-} \\
r
\end{array}\right)\right] .
\end{aligned}
$$

Recall that, in the case where all Bobs have the same value, we have $\# \mathcal{S}_{l}^{(q+1)}$ combinations to attribute the value \pm 1 to all $l$ out of $q$ Bobs. Here, the sum still has $\left(\begin{array}{l}q \\ l\end{array}\right)$ many terms, but some multiply to +1 , while others to -1 , depending on how many elements are drawn from $q_{-}$. To correctly count the numbers of combinations leading to the sign \pm 1 , we use the Chu-Vandermonde identity (A3c). The idea here is to divide the total amount of options $q$ into two subsets $q_{+}$and $q_{-}$, and then count all possible combinations to draw elements from these subsets. But due to the negativity of elements from the set $q_{-}$, we need to include a negative sign for $\left(\begin{array}{c}q_{-} \\ r\end{array}\right)$ if $r$ is odd. It is important that, due to the alternating sign, almost all terms in Eq. (A8) cancel each other. In fact, the following two relations hold:

$$
\begin{aligned}
\sum_{k=1}^{\left\lfloor\frac{n-1}{2}\right\rfloor}\left[\sum_{r=0}^{2 k-1}(-1)^{r}\left(\begin{array}{c}
q_{+} \\
2 k-1-r
\end{array}\right)\left(\begin{array}{c}
q_{-} \\
r
\end{array}\right)\right]=(-1)^{q_{-}+1} \delta_{\left\lfloor\frac{n}{2}\right\rfloor,\left\lceil\frac{n}{2}\right\rceil} \delta_{n-1, q} \quad \text { and } \\
\sum_{k=1}^{\left\lfloor\frac{n-1}{2}\right\rfloor}\left[\sum_{r=0}^{2 k}(-1)^{r}\left(\begin{array}{c}
q_{+} \\
2 k-r
\end{array}\right)\left(\begin{array}{c}
q_{-} \\
r
\end{array}\right)\right]=-1 \quad \forall n \in \mathbb{N}, q_{ \pm} \geqslant 1, \quad q_{+}+q_{-} \leqslant n-1 .
\end{aligned}
$$

Showing the validity of these relations concludes the proof, as inserting them into Eq. (A8) leads to the maximum of $\tilde{\mathcal{B}}^{(n)}=1$. To prove Eq. (A9a) we order the left-hand side of it by positive and negative contributions:

$$
\begin{aligned}
\sum_{k=1}^{\left\lfloor\frac{n-1}{2}\right\rfloor}\left[\sum_{r=0}^{2 k-1}(-1)^{r}\left(\begin{array}{c}
q_{+} \\
2 k-1-r
\end{array}\right)\left(\begin{array}{c}
q_{-} \\
r
\end{array}\right)\right]= & \sum_{k=1}^{\left\lfloor\frac{n-1}{2}\right\rfloor}\left[\sum_{r=0}^{k-1}\left(\begin{array}{c}
q_{+} \\
2 k-1-2 r
\end{array}\right)\left(\begin{array}{c}
q_{-} \\
2 r
\end{array}\right)\right] \\
& -\sum_{k=1}^{\left\lfloor\frac{n-1}{2}\right\rfloor}\left[\sum_{r=0}^{k-1}\left(\begin{array}{c}
q_{+} \\
2 k-1-(2 r+1)
\end{array}\right)\left(\begin{array}{c}
q_{-} \\
2 r+1
\end{array}\right)\right] .
\end{aligned}
$$

The idea is to use the Pascal triangle relation (A3b) to eliminate the problems that arise due to the alternating sign. Via Eq. (A3b) we thus split the right-hand side of Eq. (A10a) into the following two expressions:

$$
\begin{aligned}
& \sum_{k=1}^{\left\lfloor\frac{n-1}{2}\right\rfloor} \sum_{r=0}^{k-1}\left(\begin{array}{c}
q_{-}-1 \\
2 r
\end{array}\right)\left[\left(\begin{array}{c}
q_{+}-1 \\
2 k-1-2 r
\end{array}\right)+\left(\begin{array}{c}
q_{+}-1 \\
2(k-1)-2 r
\end{array}\right)\right]=\sum_{x=0}^{2\left\lfloor\frac{n-1}{2}\right\rfloor-1} \sum_{r=0}^{\left\lfloor\frac{x}{2}\right\rfloor}\left(\begin{array}{c}
q_{-}-1 \\
2 r
\end{array}\right)\left(\begin{array}{c}
q_{+}-1 \\
x-2 r
\end{array}\right) \text {, }
\end{aligned}
$$

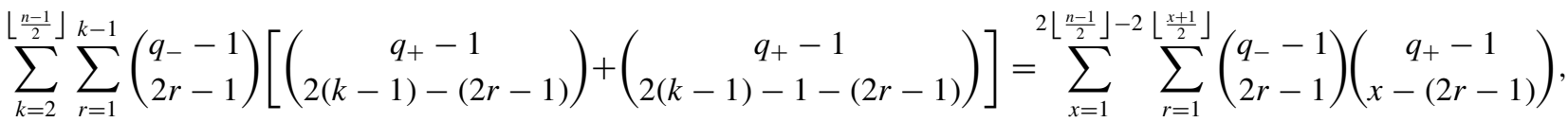

where we introduced a new index of summation $x$ to simplify both expressions. We dropped the contributions from $k=1$ and $r=0$ in Eq. (A11b), as they vanish anyway. To proceed, we add the right-hand sides of Eqs. (A11a) and (A11b). All integers from $r=0$ up to $r=x$, for all $x \in\left\{0, \ldots, 2\left\lfloor\frac{n-1}{2}\right\rfloor-2\right\}$, appear in this sum. Therefore, the right-hand side of Eq. (A10a) is given by

$$
\begin{aligned}
& \sum_{k=1}^{\left\lfloor\frac{n-1}{2}\right\rfloor}\left[\sum_{r=0}^{k-1}\left(\begin{array}{c}
q_{+} \\
2 k-1-2 r
\end{array}\right)\left(\begin{array}{c}
q_{-} \\
2 r
\end{array}\right)\right] \\
& =\sum_{x=0}^{2\left\lfloor\frac{n-1}{2}\right\rfloor-2} \sum_{y=0}^{x}\left(\begin{array}{c}
q_{-}-1 \\
y
\end{array}\right)\left(\begin{array}{c}
q_{+}-1 \\
x-y
\end{array}\right) \\
& \quad+\sum_{r=0}^{\left\lfloor\frac{n-1}{2}\right\rfloor-1}\left(\begin{array}{c}
q_{-}-1 \\
2 r
\end{array}\right)\left(\begin{array}{c}
q_{+}-1 \\
2\left\lfloor\frac{n-1}{2}\right\rfloor-1-2 r
\end{array}\right),
\end{aligned}
$$

where we used $\left\lfloor\left\lfloor\frac{n-1}{2}\right\rfloor-\frac{1}{2}\right\rfloor=\left\lfloor\frac{n-1}{2}\right\rfloor-1$. To simplify Eq. (A12), note that the second sum only yields a nontrivial contribution if $2 r \leqslant q_{-}-1$ and $2 r \geqslant 2\left\lfloor\frac{n-1}{2}\right\rfloor-q+q_{-}$, which is only possible if $q \geqslant 2\left\lfloor\frac{n-1}{2}\right\rfloor+1$. As we additionally have the constraint $q \leqslant n-1$, we require $q=n-1$ and $n$ needs to be an even integer. In this case, the only nonvanishing term in the second sum in Eq. (A12) is a single expression equal to +1 , corresponding to $r=\frac{q_{-}-1}{2}$, which can only be a valid integer if $q_{-}$is odd. Beyond this, we use the ChuVandermonde identity (A3c) to simplify the first expression of the right-hand side in Eq. (A12) and obtain

$$
\begin{aligned}
& \sum_{k=1}^{\left\lfloor\frac{n-1}{2}\right\rfloor}\left[\sum_{r=0}^{k-1}\left(\begin{array}{c}
q_{+} \\
2 k-1-2 r
\end{array}\right)\left(\begin{array}{c}
q_{-} \\
2 r
\end{array}\right)\right] \\
& =\sum_{x=0}^{2\left\lfloor\frac{n-1}{2}\right\rfloor-2}\left(\begin{array}{c}
q-2 \\
x
\end{array}\right)+\delta_{n-1, q} \delta_{\left\lfloor\frac{n}{2}\right\rfloor,\left\lceil\frac{n}{2}\right\rceil_{\left\lfloor\frac{q_{-}-1}{2}\right\rfloor, \frac{q_{-}-1}{2}}}
\end{aligned}
$$


The same procedure can be applied to the right-hand side of Eq. (A10b). Ultimately, it leads to

$$
\begin{aligned}
& \sum_{k=1}^{\left\lfloor\frac{n-1}{2}\right\rfloor}\left[\sum_{r=0}^{k-1}\left(\begin{array}{c}
q_{+} \\
2 k-1-(2 r+1)
\end{array}\right)\left(\begin{array}{c}
q_{-} \\
2 r+1
\end{array}\right)\right] \\
& =\sum_{x=0}^{2\left\lfloor\frac{n-1}{2}\right\rfloor-2}\left(\begin{array}{c}
q-2 \\
x
\end{array}\right)+\delta_{n-1, q} \delta_{\left\lfloor\frac{n}{2}\right\rfloor,\left\lceil\frac{n}{2}\right\rceil} \delta_{\left\lfloor\frac{q_{-}}{2}\right\rfloor, \frac{q_{-}}{2}},
\end{aligned}
$$

where the additional contribution is now only obtained if $q_{-}$is an even integer. The difference between Eqs. (A13) and (A14) represents the left-hand side of Eq. (A10a). We thus obtain

$$
\begin{aligned}
& \sum_{k=1}^{\left\lfloor\frac{n-1}{2}\right\rfloor}\left[\sum_{r=0}^{2 k-1}(-1)^{r}\left(\begin{array}{c}
q_{+} \\
2 k-1-r
\end{array}\right)\left(\begin{array}{c}
q_{-} \\
r
\end{array}\right)\right] \\
& =\delta_{n-1, q} \delta_{\left\lfloor\frac{n}{2}\right\rfloor,\left\lceil\frac{n}{2}\right\rceil}\left(\delta_{\left\lfloor\frac{q_{-}-1}{2}\right\rfloor, \frac{q_{-}-1}{2}}-\delta_{\left\lfloor\frac{q_{-}}{2}\right\rfloor, \frac{q_{-}}{2}}\right) \\
& =(-1)^{q_{-}+1} \delta_{n-1, q} \delta_{\left\lfloor\frac{n}{2}\right\rfloor,\left\lceil\frac{n}{2}\right\rceil},
\end{aligned}
$$

which proves identity (A9a). Essentially the same approach now leads to the proof of relation (A9b). Only minor and straightforward adjustments for the index of summations are needed, which then leads to

$$
\begin{aligned}
& \sum_{k=1}^{\left\lfloor\frac{n-1}{2}\right\rfloor}\left[\sum_{r=0}^{2 k}(-1)^{r}\left(\begin{array}{c}
q_{+} \\
2 k-r
\end{array}\right)\left(\begin{array}{c}
q_{-} \\
r
\end{array}\right)\right] \\
& =-\left(\begin{array}{c}
q-2 \\
0
\end{array}\right)+\left(\begin{array}{c}
q-2 \\
2\left\lfloor\frac{n-1}{2}\right\rfloor-1
\end{array}\right)-\delta_{n-1, q} \delta_{\left\lfloor\frac{n}{2}\right\rfloor,\left\lceil\frac{n}{2}\right\rceil}=-1,
\end{aligned}
$$

because the second binomial coefficient is +1 if $n$ is even and $q=n-1$, and zero otherwise. This concludes the proof.

\section{APPENDIX B: THE SUBCLASS OF PARITY-CHSH INEQUALITIES}

The Bell inequality (A1) is constructed around two central restrictions we impose on the Bell setting. First, we want to achieve a large Bell value if the quantum resource is given by an $n$-GHZ state, and second, this Bell value is achievable if Alice measures $A_{0}=\sigma_{z}$. As the Bell inequality is tested for violation in a DIQKD protocol, these restrictions are clearly motivated by Theorem 1 of Ref. [4], which states that maximum correlation among all $n$ parties with a GHZ state requires all parties to measure $\sigma_{z}$.

A priori, it is not clear how to devise a useful Bell inequality that is particularly well suited for the $n$-GHZ state. The MABK inequality [21-23] for instance allows a maximum violation by the $n$-GHZ state, as discussed in Ref. [39]. For DIQKD, however, the MABK inequality is not suitable because the very structure of it prohibits to simultaneously achieve perfectly correlated measurement results among all parties and sufficiently high Bell-inequality violation (see Ref. [25] for details). Imposing the additional constraint on the Bell setting, that Alice should in principle be able to measure $A_{0}=\sigma_{z}$ without compromising the possibility to violate the Bell inequality, has led us to our inequality (A1). The parity-
CHSH inequality [18],

$$
\begin{aligned}
\mathcal{B}_{\text {Parity }}^{(n)}:= & \left\langle A_{1} \otimes \frac{B_{0}^{(2)}+B_{1}^{(2)}}{2} \bigotimes_{j=3}^{n} B^{(j)}\right\rangle \\
& -\left\langle A_{0} \otimes \frac{B_{0}^{(2)}-B_{1}^{(2)}}{2}\right\rangle \leqslant 1 \leqslant \sqrt{2},
\end{aligned}
$$

also embraces this idea. Here, each $\mathrm{Bob}^{(j)}$ for $j \geqslant 3$ only has one observable. The parity-CHSH inequality is a special case of our Bell inequality (A1), which follows for $B_{0}^{(j)}=B_{1}^{(j)}$ for all $j \geqslant 3$ and therefore $B_{-}^{(j)}=0$ and $B_{+}^{(j)}=B_{0}^{(j)}=: B^{(j)}$.

\section{APPENDIX C: OPTIMAL MEASUREMENTS AND BELL VALUE}

As our main goal was to establish a useful Bell inequality for multipartite DIQKD, our focus is not the complete characterization of our Bell inequality. For completeness, however, we want to address some properties; in particular, we suggest measurement observables for all parties that lead to a maximum Bell value if the $n$-GHZ state is measured, as this is relevant for QKD. To motivate the optimal choices for the observables given the GHZ state is measured, recall that a general qubit observable can be parametrized as

$$
\begin{aligned}
B_{i}^{(j)}= & \cos \left(\varphi_{i}^{(j)}\right) \sin \left(\theta_{i}^{(j)}\right) \sigma_{x} \\
& +\sin \left(\varphi_{i}^{(j)}\right) \sin \left(\theta_{i}^{(j)}\right) \sigma_{y}+\cos \left(\theta_{i}^{(j)}\right) \sigma_{z},
\end{aligned}
$$

and analogously for $A_{1}$. Note that $B_{0}^{(j)}, B_{1}^{(j)}$ always appear as $B_{-}^{(j)} \propto B_{0}^{(j)}-B_{1}^{(j)}$ in our Bell inequality, if paired with $A_{0}$ or if no observable of Alice is included. To maximize the corresponding expectation values, it is best to eliminate the contribution of all $B_{-}^{(j)}$ in the $\sigma_{x}$ and $\sigma_{y}$ directions, as this part vanishes anyway due to the structure of the stabilizer representation of the GHZ state (see main text). This translates to $\varphi_{0}^{(j)}=\varphi_{1}^{(j)}$ for all $j \in[n]$, as a necessary condition to guarantee $B_{-} \propto \sigma_{z}$. Likewise, the expression $B_{+}^{(j)} \propto B_{0}^{(j)}+B_{1}^{(j)}$ appears only in combination with $A_{1}$. Because all operators with $s_{1}=1$ in the stabilizer representation of the GHZ state have full weight, we might as well take that $A_{1}$ and all $B_{+}^{(j)}$ expressions have no contribution in the $\sigma_{z}$ direction, to gain a large contribution to the Bell value from $\left\langle A_{1} \bigotimes_{j=2}^{n} B_{+}^{(j)}\right\rangle$. Due to $\cos (\alpha)=-\cos (\pi \pm \alpha)$, we extract $\theta_{1}^{(j)}=\pi \pm \theta_{0}^{(j)}$ for all $j \in[n]$ from the representation (C1), as a necessary condition to eliminate the $\sigma_{z}$ contribution of $B_{+}^{(j)}$. Beyond that, we note that $\sin (\pi \pm \alpha)=\mp \sin (\alpha)$. Together with $\varphi_{0}^{(j)}=\varphi_{1}^{(j)}$, the choice $\theta_{1}^{(j)}=\pi+\theta_{0}^{(j)}$ eliminates $B_{+}^{(j)}$, which is why we use $\theta_{1}^{(j)}=\pi-\theta_{0}^{(j)}$ in the following. Finally, we numerically find that for a given choice of $A_{1}$, the actual value of the azimuthal angle $\varphi_{0}^{(j)}, \varphi_{1}^{(j)}$ is irrelevant for maximizing the Bell value, as long as they are equal for each Bob. Therefore, we set $\varphi_{0}^{(j)}=$ $\varphi_{1}^{(j)}=0$ for all $j \in[n]$ and $\varphi_{A_{1}}=0$. Furthermore, the polar angles $\theta_{0}^{(j)}, \theta_{1}^{(j)}$ can be chosen the same for every Bob, without compromising the possibility to achieve the maximum Bell value. We therefore set $\theta_{0}^{(j)}=\theta$ and $\theta_{1}^{(j)}=\pi-\theta$ for all $j \in$ [n]. In total, the maximum Bell value $\mathcal{B}^{(n)}$, given an $n$-GHZ 
state is measured, can be achieved with

$$
\begin{aligned}
A_{0} & =\sigma_{z}, \quad A_{1}=\sigma_{x}, \quad B_{0}^{(j)}=\sin (\theta) \sigma_{x}+\cos (\theta) \sigma_{z}, \\
B_{1}^{(j)} & =\sin (\theta) \sigma_{x}-\cos (\theta) \sigma_{z} \quad \forall j \in[n],
\end{aligned}
$$

where the optimal value of the polar angle $\theta$ depends on the number of parties, $n$. This choice allows a straightforward calculation of the Bell value with the $n$-GHZ state

$$
\begin{aligned}
g_{\mathrm{GHZ}}^{(n)}= & {\left[\sin (\theta)^{n-1}-\delta_{\left\lfloor\frac{n}{2}\right\rfloor,\left\lceil\frac{n}{2}\right\rceil} \cos (\theta)^{n-1}\right] } \\
& -\sum_{k=1}^{\left\lfloor\frac{n-1}{2}\right\rfloor} \cos (\theta)^{2 k-1}\left[\left(\begin{array}{c}
n-1 \\
2 k-1
\end{array}\right)+\cos (\theta)\left(\begin{array}{c}
n-1 \\
2 k
\end{array}\right)\right],
\end{aligned}
$$

which can be simplified to the set of Eqs. (14).
[1] M. F. Riedel, D. Binosi, R. Thew, and T. Calarco, Quantum Sci. Technol. 2, 030501 (2017).

[2] A. Acín, I. Bloch, H. Buhrman, T. Calarco, C. Eichler, J. Eisert, D. Esteve, N. Gisin, S. J. Glaser, F. Jelezko et al., New J. Phys. 20, 080201 (2018).

[3] S. Wehner, D. Elkouss, and R. Hanson, Science 362, eaam9288 (2018).

[4] M. Epping, H. Kampermann, C. Macchiavello, and D. Bruß, New J. Phys. 19, 093012 (2017).

[5] C. H. Bennett and G. Brassard, in Proceedings of the IEEE International Conference on Computers, Systems and Signal Processing (IEEE, New York, 1984), pp. 175-179.

[6] A. K. Ekert, Phys. Rev. Lett. 67, 661 (1991).

[7] D. Bruß, Phys. Rev. Lett. 81, 3018 (1998).

[8] D. Mayers and A. Yao, in Proceedings of the 39th Annual Symposium on Foundations of Computer Science (IEEE Computer Society, Washington, DC, 1998), pp. 503-509.

[9] J. Barrett, L. Hardy, and A. Kent, Phys. Rev. Lett. 95, 010503 (2005).

[10] R. Colbeck, arXiv:0911.3814.

[11] A. Acín, N. Brunner, N. Gisin, S. Massar, S. Pironio, and V. Scarani, Phys. Rev. Lett. 98, 230501 (2007).

[12] S. Pironio, A. Acín, N. Brunner, N. Gisin, S. Massar, and V. Scarani, New J. Phys. 11, 045021 (2009).

[13] L. Masanes, S. Pironio, and A. Acín, Nat. Commun. 2, 238 (2011).

[14] C. A. Miller and Y. Shi, SIAM J. Comput. 46, 1304 (2017).

[15] U. Vazirani and T. Vidick, Phys. Rev. Lett. 113, 140501 (2014).

[16] R. Arnon-Friedman, R. Renner, and T. Vidick, SIAM J. Comput. 48, 181 (2019).

[17] R. Arnon-Friedman, F. Dupuis, O. Fawzi, R. Renner, and T. Vidick, Nat. Commun. 9, 459 (2018).

[18] J. Ribeiro, G. Murta, and S. Wehner, Phys. Rev. A 100, 026302 (2019).
[19] J. F. Clauser, M. A. Horne, A. Shimony, and R. A. Holt, Phys. Rev. Lett. 23, 880 (1969).

[20] D. M. Greenberger, M. A. Horne, and A. Zeilinger, in Bell's Theorem, Quantum Theory and Conceptions of the Universe (Springer, Berlin, 1989), pp. 69-72.

[21] N. D. Mermin, Phys. Rev. Lett. 65, 1838 (1990).

[22] M. Ardehali, Phys. Rev. A 46, 5375 (1992).

[23] A. V. Belinskiı̌ and D. N. Klyshko, Phys. Usp. 36, 653 (1993).

[24] R. Augusiak, A. Salavrakos, J. Tura, and A. Acín, New J. Phys. 21, 113001 (2019).

[25] T. Holz, D. Miller, H. Kampermann, and D. Bruß, Phys. Rev. A 100, 026301 (2019).

[26] M. Navascués, S. Pironio, and A. Acín, Phys. Rev. Lett. 98, 010401 (2007).

[27] M. Navascués, S. Pironio, and A. Acín, New J. Phys. 10, 073013 (2008).

[28] B. S. Cirel'son, Lett. Math. Phys. 4, 93 (1980).

[29] A. Salavrakos, R. Augusiak, J. Tura, P. Wittek, A. Acín, and S. Pironio, Phys. Rev. Lett. 119, 040402 (2017).

[30] D. Gottesman, arXiv:quant-ph/9705052.

[31] M. Hein, W. Dür, J. Eisert, R. Raussendorf, M. Nest, and H.-J. Briegel, in Entanglement in Graph States and Its Applications, Proceedings of the International School of Physics "Enrico Fermi” (IOS Press, Amsterdam, 2005), Vol. 162, pp. 115-218.

[32] G. Svetlichny, Phys. Rev. D 35, 3066 (1987).

[33] P. Wittek, ACM Trans. Math. Softw. 41, 21 (2015).

[34] R. Konig, R. Renner, and C. Schaffner, IEEE Trans. Inf. Theory 55, 4337 (2009).

[35] E. Y.-Z. Tan, R. Schwonnek, K. T. Goh, I. W. Primaatmaja, and C. C.-W. Lim, arXiv:1908.11372.

[36] J. Ribeiro, G. Murta, and S. Wehner, Phys. Rev. A 97, 022307 (2018).

[37] J. Briët and P. Harremoës, Phys. Rev. A 79, 052311 (2009).

[38] R. F. Werner and M. M. Wolf, Phys. Rev. A 61, 062102 (2000).

[39] R. F. Werner and M. M. Wolf, Phys. Rev. A 64, 032112 (2001). 